\title{
CORRELATION OF X-RAY AND INFRARED EMISSION OF RADIO-QUIET QSOs
}

\author{
D. M. Worrall \\ Harvard-Smithsonian Center for Astrophysics, Cambridge, MA, U.S.A.
}

\begin{abstract}
A correlation of the form $l_{x} \propto l_{i r}^{\gamma}$, with $\gamma=0.83$, is found between $2 \mathrm{keV}$ monochromatic X-ray luminosity and $1.65 \mu \mathrm{m}$ monochromatic infrared luminosity for a sample of optically selected radio-quiet QSOs. This is equivalent to an average $1.65 \mu \mathrm{m}$ to $2 \mathrm{keV}$ spectral index of $\alpha_{i r / x}=1.28+0.05 \log \left(l_{i r} / 10^{31} \mathrm{ergs} \mathrm{s}^{-1} \mathrm{~Hz}^{-1}\right)$. The ratio of X-ray to infrared luminosity decreases with increasing infrared luminosity in a manner similar to the relationship between Xray and optical luminosity. The standard deviations of the distributions of data about the best-fit functions are comparable for the X-ray versus infrared and X-ray versus optical fits. Thus, contrary to a previous claim, the infrared luminosity is not better than the optical luminosity at predicting the X-ray emission from QSOs.
\end{abstract}

\section{INTRODUCTION}

There is general agreement that optically selected QSOs exhibit a dependence of monochromatic $2 \mathrm{keV}$ X-ray luminosity, $l_{x}$, on monochromatic $2500 \AA$ luminosity, $l_{o}$, of the form $l_{x} \propto l_{o}^{\alpha}$, with $\alpha$ in the range 0.6-0.9 (e.g., Avni and Tananbaum 1982, 1986; Kriss and Canizares 1985). At a rest wavelength of $2500 \AA$, QSO spectra are generally flat and may be dominated by thermal emission from an accretion disk (e.g., Shields 1978; Malkan and Sargent 1982). However, the near-infrared $(1.65 \mu \mathrm{m})$ radiation is dominated by a steeper component, which generally fits a power law of energy index $\alpha_{i r} \gtrsim 1.0$ and often extrapolates well to X-ray emission at $\sim 2 \mathrm{keV}$ (e.g., Malkan and Sargent 1982; Elvis et al. 1986). Malkan (1984) reports that for Seyfert 1 galaxies and QSOs, $\mathrm{X}$-ray and near-infrared luminosity exhibit a much tighter correlation than any other correlations between continuum bands. Here this issue is examined by comparing the X-ray and near-infrared correlation with the X-ray and $2500 \AA$ correlation for a carefully selected sample of radio-quiet QSOs.

\section{SAMPLE}

The sample has been extracted from the optically selected sample of Avni and Tananbaum (1986). Objects with a spectral index between $5 \mathrm{GHz}$ and $2500 \AA$ greater than 0.35 have been deleted because their X-ray emission is enhanced by some process involving their radio emission (e.g., $\mathrm{Ku}$, Helfand and Lucy 1980; Worrall et al. 1986). Of the 134 remaining objects, 69 have near-infrared photometric measurements. Objects in the Palomar Bright Quasar Survey account for $\sim 3 / 4$ of these, and their infrared measurements are given by Neugebauer et al. (1986). Data for the 
other QSOs are from a variety of published sources. Optical and Einstein Observatory Imaging Proportional Counter X-ray data are as given in Avni and Tananbaum (1986) and Tananbaum et al. (1986), except that the X-ray luminosities are corrected to values appropriate for an X-ray spectral index of 1.0 rather than 0.5 (see Wilkes and Elvis 1986). Worrall (1986) gives a full description of the data.

\section{RESULTS}

Figure 1 shows separate plots of monochromatic X-ray luminosity versus optical luminosity and infrared luminosity. From visual inspection, the standard deviation of the data around the regression line appears to be roughly the same for both plots. This has been confirmed by fitting functions of the form $l_{x} / 10^{27}=A\left(l_{o} / 10^{31}\right)^{\alpha}$ and $l_{x} / 10^{27}=C\left(l_{i r} / 10^{31}\right)^{\gamma}$ using the fitting method of Avni et al. (1980), which treats both detections and upper limits. In this method, the distribution of residuals around the best-fit function is assumed to be Gaussian, and the standard deviation of the distribution, $\sigma_{G}$ is treated as a free parameter. Taking only the data shown as filled symbols in Figure 1, the values found for $\sigma_{G}$ are $0.38 \pm 0.06$ for the upper graph and $0.41 \pm 0.08$ for the lower graph (90\% confidence errors for one interesting parameter). The values increase to $0.50 \pm$ 0.08 and $0.52 \pm 0.08$, respectively, if all the data are fitted. These results are contrary to those of Malkan (1984), and imply that the infrared luminosity is not significantly better than the optical luminosity as an estimator of the X-ray emission from these QSOs. The fit to the upper graph gives $\log A=-0.11 \pm 0.08$ and $\alpha=0.79 \pm 0.08$, consistent with previous results. The lower graph gives $\log C=-0.40 \pm 0.11$ and $\gamma=0.83 \pm 0.12$. (All errors are given as the projection of the $90 \%$ confidence error surface for one interesting parameter onto the axis for that parameter.)

The ratio of X-ray to infrared luminosity decreases with increasing infrared luminosity in a manner similar to the relationship between $\mathrm{X}$-ray and optical luminosity. The result for the Xray versus infrared correlation is equivalent to an average $1.65 \mu \mathrm{m}$ to $2 \mathrm{keV}$ spectral index of $\alpha_{i r / x}=1.28+0.05 \log \left(l_{i r} / 10^{31}\right)$.
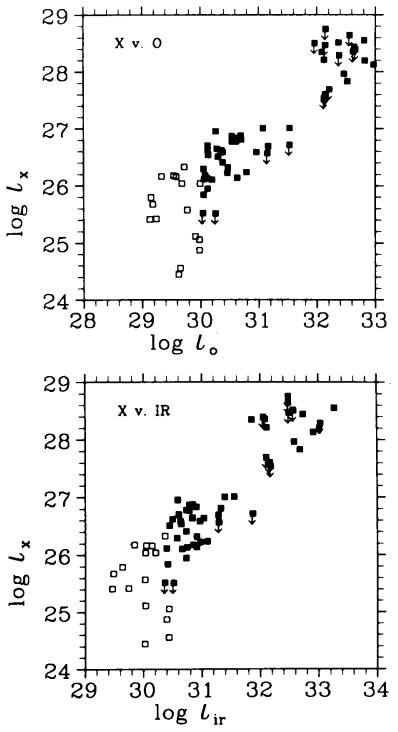

Figure 1. Logarithmic plots of monochromatic Xray luminosity at $2 \mathrm{keV}$ versus monochromatic optical luminosity at $2500 \AA$ (above) and monochromatic infrared luminosity at $1.65 \mu \mathrm{m}$ (below). Luminosities are in units of ergs $\mathrm{s}^{-1} \mathrm{~Hz}^{-1}$ for the source frame and have been calculated assuming a Friedmann cosmology with $H_{o}=50$ $\mathrm{km} \mathrm{s}^{-1} \mathrm{Mpc}^{-1}$ and $q_{o}=0$. Objects for which the monochromatic optical luminosity is $<10^{30} \mathrm{ergs} \mathrm{s}^{-1} \mathrm{~Hz}^{-1}$ are shown by open symbols: these data show the greatest scatter, attributed to the larger and more uncertain X-ray absorptions at low luminosities (Zamorani 1986). 
I am grateful to G. Neugebauer for providing an early preprint of Neugebauer et al. (1986). This work was supported by NASA Contract NAS8-30751 and Smithsonian Institutional funds.

\section{REFERENCES}

Avni, Y., Soltan, A., Tananbaum, H., and Zamorani, G. 1980, Ap.J., 238, 800.

Avni, Y., and Tananbaum, H. 1982, Ap.J. (Letters), 262, L17.

Avni, Y., and Tananbaum, H. 1986, Ap.J., 305, 83.

Elvis, M., Green, R.F., Bechtold, J., Schmidt, M., Neugebauer, G., Soifer, B.T., Matthews, K., and Fabbiano, G. 1986, Ap.J., 310, in press.

Kriss, G.A., and Canizares, C.R. 1985, Ap.J., 297, 177.

Ku, W.H-M., Helfand, D.J., and Lucy, L.B. 1980, Nature, 288, 323.

Malkan, M.A. 1984, X-ray and UV Emission from Active Galactic Nuclei, ed. J. Trümper and W. Brinkmann (Garching: Max-Planck-Institut), p121.

Malkan, M.A., and Sargent, W.L.W. 1982, Ap.J., 254, 22.

Neugebauer, G., Green, R.F., Matthews, K., Schmidt, M., Soifer, B.T., and Bennett, J. 1986, Ap.J. Suppl., in press.

Shields, G. 1978, Nature, 272, 706.

Tananbaum, H., Avni, Y., Green, R.F., Schmidt, M., and Zamorani, G. 1986, Ap.J., 305, 57.

Wilkes, B.J., and Elvis, M. 1986, Ap.J., submitted.

Worrall, D.M. 1986, Ap.J., submitted.

Worrall, D.M., Giommi, P., Tananbaum, H., and Zamorani, G. 1986, Ap.J., in press.

Zamorani, G. 1986, Quasars, eds. G. Swarup and V.K. Kapahi (Reidel), p223. 